\title{
Contribution du manioc à l'alimentation et à la nutrition des enfants dans la commune de Djidja au Bénin
}

\author{
H. H. SAHOUEGNON ${ }^{*}$, E. C. MITCHIKPE, A. P. P. KAYODE et R. A. M. DOSSA \\ Département de Nutrition et de Sciences Alimentaires Faculté des Sciences Agronomiques, \\ Université d'Abomey-Calavi, 01 BP 526 Cotonou, Bénin. \\ *Auteur correspondant, E-mail: hyanefr@yahoo.fr ; Tel : +22966622206.
}

\section{RESUME}

La présente étude vise à évaluer la contribution des aliments à base de manioc à l'alimentation et à la nutrition des enfants de 6 à 35 mois de la commune de Djidja. En outre, les facteurs socioéconomiques et culturels qui influencent la consommation desdits produits ont été identifiés. L'étude s'est déroulée en deux phases. La première phase a consisté en une enquête de consommation alimentaire et nutritionnelle au cours de laquelle les données de consommation alimentaire et nutritionnelle ont été collectées sur un échantillon de 106 enfants réparti en trois tranches d'âge. Dans la deuxième phase, les données socioéconomiques et culturelles ont été collectées à travers des focus group auprès de 400 acteurs (producteurs, transformatrices et mères d'enfants). La prévalence des différents types de malnutrition obtenue se présente comme suit: émaciation : $8,6 \%$; retard de croissance: $32,7 \%$ et insuffisance pondérale : $17,1 \%$. Il en découle que la situation nutritionnelle des enfants de 6 à 35 mois pose un sérieux problème de santé publique dans la commune de Djidja. Le dérivé du manioc le plus consommé par les enfants de 6 à 35 mois est le gari. Sa contribution aux taux de couverture des besoins en nutriments par les aliments de complément et l'alimentation des enfants de 6 à 35 mois est relativement faible. Elle varie de 4 à $22 \%$ pour l'énergie, 2 à $14 \%$ pour les protéines, 1 à $9 \%$ pour le fer et 1 à $5 \%$ pour le zinc dans les trois tranches d'âge. Par ailleurs, les facteurs socioéconomiques et culturels qui influencent la consommation des dérivés du manioc par les enfants sont le revenu des ménages, la commodité (la disponibilité locale et l'accessibilité permanente du gari, font qu'il est plus facile aux mères de le donner aux enfants pour calmer leur faim), la disponibilité et l'accessibilité permanente des dérivés de manioc et la facilité de préparation.

(c) 2014 International Formulae Group. All rights reserved.

Mots clés : Manioc, malnutrition, gari, enfants, facteurs socioéconomiques.

\section{INTRODUCTION}

La malnutrition par carence en micronutriments constitue un problème de santé publique important en Afrique subsaharienne. Selon les résultats de l'Analyse Globale de la Vulnérabilité, de la Sécurité Alimentaire et de la Nutrition au Bénin (PAM, 2009) le retard de croissance (malnutrition chronique) touche $37 \%$ des enfants de 6 à 59 mois dont $12 \%$ sous forme sévère. L'émaciation (malnutrition aigüe) touche $5 \%$ des enfants dont $1 \%$ sous la forme sévère et $17 \%$ des enfants souffrent d'insuffisance pondérale dont $3 \%$ sous forme sévère. Les conséquences de la malnutrition sont nombreuses et diverses. Elle entrave le 
développement physique et intellectuel de l'individu, diminue la résistance de l'organisme aux maladies, entraînant la baisse de la productivité (FAO, 2001). Pour sa réduction, de grandes stratégies telles que la supplémentation, la fortification des aliments et la diversification alimentaire ont été proposées et expérimentées. Mais ces différentes stratégies se heurtent à de nombreuses difficultés, fragilisant leur durabilité. Face à toutes ces difficultés, d'autres approches alimentaires telles que la biofortification et l'amélioration des procédés de transformation sont proposées en vue d'alléger le problème de la malnutrition de façon durable. La biofortification est une nouvelle approche consistant à augmenter au champ la teneur en micronutriments des cultures de subsistance dont se nourrissent les populations pauvres (Harvestplus, 2009). Cette stratégie s'appuie sur le constat que l'alimentation de base de ces populations est essentiellement constituée d'aliments riches en glucides. Le manioc fait partie de ces aliments riches en glucides consommés par ces populations. Cependant, sur le plan nutritionnel, le manioc est pauvre en micronutriments. Ainsi différents aliments de base sont biofortifiés en divers nutriments dont le manioc biofortifié en $\beta$ - carotène. La présente étude s'inscrit dans le projet INSTAPA (Improved Nutrition Through Staple Food in Africa). Dans le cadre de ce projet, il est envisagé l'introduction d'une variété de manioc biofortifiée en vitamine $\mathrm{A}$ auprès des communautés du Bénin. Cependant, pour que cette stratégie de lutte contre la carence en vitamine A soit effective, il est important de connaître la place et l'importance du manioc dans l'alimentation de ces populations. Au Bénin, plusieurs études ont été réalisées sur le manioc. Parmi ces études, on distingue celles portant sur les modes de cuisson des racines de manioc (Nago et Hounhouigan, 1998) ; d'autres sur la production du «lafou», un produit fermenté dérivé du manioc (Chabi China, 2000). Des études se sont également intéressées aux caractéristiques physico-chimiques des principales variétés de manioc en usage au Bénin (Adjadogbedji, 2000). Les caractéristiques chimiques, physiques et microbiologiques du «lafou», produit au Bénin, ont été étudiées (Padonou et al., 2009). Cependant, ces études n'ont pas rapporté la contribution des produits à base de manioc à l'alimentation et à la nutrition des enfants de 6 à 35 mois. La présente étude vise à apprécier la contribution des aliments à base de manioc à l'alimentation et la nutrition des enfants et d'identifier les facteurs socioéconomiques et culturels influençant cette consommation. De façon spécifique, cette étude se propose de (i) apprécier l'état nutritionnel des enfants de 6 à 35 mois; (ii) évaluer la contribution du manioc aux apports en énergie et en nutriments des enfants de 6 à 35 mois et ; (iii) identifier les facteurs socioéconomiques et culturels qui influencent la consommation des produits à base de manioc par les enfants de 6 à 35 mois.

\section{MATERIEL ET METHODES Milieu d'étude}

L'étude a été conduite dans la commune de Djidja, (considérée comme le grenier du département de Zou) située au centre du Bénin. Elle est la plus vaste commune du département du zou avec une superficie de $2184 \mathrm{~km}^{2}$. Cette commune est subdivisée en 79 villages regroupés en 12 arrondissements. On y rencontre par endroit, des îlots de forêt donnant l'aspect d'une végétation arborée ou arbustive. La commune de Djidja est considérée comme le grenier dudit département, divisée en quatre zones telles que les zones du Sud, du Nord, du Centre et de l'Est ; trois parmi elles (les zones du Centre, du Nord et de l'Est) représentent les grandes zones de production agricole de la commune. De plus, les statistiques montrent que dans ce département, la commune de Djidja est le premier producteur de céréales (14973 tonnes), de tubercules (160117 tonnes) 
et de légumineuses (8357 tonnes) (ONASA /CeRPA, 2008).

\section{Echantillonnage des sujets d'enquête}

Cinq des 12 arrondissements qui composent la commune de Djidja ont été choisis de façon aléatoire dans trois différentes zones de la commune (la zone du Sud, la zone du Centre et la zone du Nord) en utilisant la méthode de tirage sans remise. Il s'agit des arrondissements de de Mougnon, de Dohouime (zone du Sud), de Djidja-centre (zone du Centre) et de Monsourou, de Outo (zone du Nord) $\left(\mathrm{RGPH}_{3}, 2004\right)$. Un effectif de 106 enfants âgés de 6 à 35 mois a été échantillonné. Cet effectif a été calculé selon la formule de la distribution normale et ses principes. Sur la base de la taille de la population des enfants de zéro à cinq ans par zone retenue, un certain nombre de ménages a été sélectionné dans chacune de ces zones. Ainsi, il a été retenu 58 ménages au Nord, 26 au Sud et 22 au Centre de la commune. Dans chaque localité choisie, la méthode d'échantillonnage de la bouteille vide décrite par Gibson (1999) a été appliquée afin de sélectionner le nombre de ménages retenus par zone. Les enfants ont été sélectionnés sur la base des critères d'éligibilité tels que l'âge compris entre 6 et 35 mois et la consommation par l'enfant d'aliments de complément. Dans une maison regroupant plusieurs ménages, un seul ménage a été sélectionné de façon aléatoire. S'il n'y a pas d'enfant éligible dans le ménage, le ménage suivant est considéré. Dans les ménages comportant des jumeaux, un seul des enfants a été choisi au hasard par tirage au sort.

\section{Collecte des données anthropométriques}

Les données collectées aux cours de cette enquête concernent les mesures anthropométriques (le poids et la taille) et les données de consommation alimentaire. Les mesures anthropométriques ont été prises afin d'apprécier l'état nutritionnel des enfants. L'âge a été déterminé afin de permettre le calcul des indicateurs de l'état nutritionnel. Le poids a été mesuré en utilisant une balance électronique pèse-personne de type SECA (seca bella 840 / bellissima 841 , précision \pm $0.5 \mathrm{~g}$ ) et une balance électronique pèse enfant de type seca (seca lena 354/364, précision $\pm 30 \mathrm{~g}$ ) posée sur une surface stable et plane. La taille des enfants de plus de deux ans a été mesurée en utilisant une microtoise (de précision $0,1 \mathrm{~cm}$ ) accrochée verticalement au mur. Pour le nourrisson ne pouvant pas encore se maintenir debout, la mesure a été prise avec une toise horizontale (Infantomètre, de précision $0,1 \mathrm{~cm}$ ) en position couchée. Ces mesures ont été prises en trois répétitions chacune. A la fin, une valeur moyenne a été calculée et retenue pour chacune d'elle.

\section{Collecte des données de consommation alimentaire}

Les données de consommation alimentaire ont été collectées par la méthode d'observation avec pesée directe des aliments. L'enquête s'est déroulée pendant une journée pour chaque enfant. Les aliments consommés par l'enfant au cours de la journée d'enquête ont été pesés à l'aide d'une balance pèse aliment de cuisine (portée : $5000 \mathrm{~g}$; précision: $1 \mathrm{~g})$. Pour cela, l'enquêteur a passé toute la journée dans le ménage (du lever au coucher de l'enfant, soit environ de 6 h à 20 h) muni d'une fiche d'observation et de pesée qui a été remplie avec l'aide des mères d'enfants. Il a également suivi la préparation des plats et a pesé tous les ingrédients entrant dans leur composition ainsi que la quantité du plat cuit.

\section{Enquête socioéconomique et culturelle}

Cette enquête s'est déroulée dans sept arrondissements sélectionnés de façon aléatoire dans les quatre zones de la commune. Sur la base des critères comme: l'accessibilité des villages, nombre de villages, et existence de groupement de transformation du manioc, 12 villages ont été sélectionnés dans l'ensemble des sept arrondissements retenus. Sur l'ensemble des 
12 villages retenus, 400 informateurs représentant les différents types d'acteurs concernés de la commune ont été représentés. Il s'agit des producteurs de manioc (109), des transformatrices du manioc (140) et des mères d'enfants d'âge compris entre 6 et 35 mois (151). Par village, nous avons réalisé un focus group par acteur. Ainsi, une enquête qualitative basée sur les techniques de la Méthode Accélérée de Recherche Participative (MARP) a été réalisée privilégiant l'outil focus group. Le guide d'entretien s'est articulé autour des aspects tels que: la production du manioc, sa transformation et sa consommation par les enfants. Les données collectées concernent: les dérivés de manioc produits dans le milieu; les différents dérivés du manioc et leur utilisation dans l'alimentation des enfants de 6 à 35 mois d'âge; les interdits alimentaires des enfants de 6 à 35 mois dans le milieu; les facteurs de motivation au changement des habitudes alimentaires.

\section{Traitement et analyse des données Données anthropométriques}

Les données anthropométriques et l'âge ont été utilisés pour calculer les indices anthropométriques. Ces indices calculés à l'aide du logiciel WHO (WHO/ANTHRO, 2006) sont exprimés en Z-score afin d'apprécier l'état nutritionnel des enfants. L'apurement relatif aux mesures anthropométriques a été fait à partir de la distribution des Z-scores obtenus avec le logiciel Anthro. Ainsi certains individus ont été écartés. La prévalence des différents types de malnutrition ainsi que les moyennes de Zscore présentées dans la partie résultat de ce document ont donc été calculées sur un échantillon de 105 sujets pour les indices Poids-Taille, et Poids-Age, et 104 sujets pour l'indice Taille-Age.

Données de consommation alimentaire

Afin d'analyser les données de l'enquête de consommation alimentaire, une base de données de consommation alimentaire et une table de composition des aliments ont été élaborées. Ensuite la base de données de consommation alimentaire a été apurée et les informations ont été entrées dans le logiciel Word Food Dietary Assessment System.

Elaboration de la base de données de consommation

A partir des données de l'enquête de consommation alimentaire, une base de données Excel a été constituée à l'aide du logiciel Microsoft Office 2007. Elle renferme les codes d'identification de chaque enfant, les quantités quotidiennes d'aliments ingérées et les quantités d'ingrédients correspondantes.

\section{Elaboration de la table de composition des aliments}

La table de composition des aliments a été utilisée pour calculer les apports en énergie et en nutriments des aliments ingérés par les enfants. Pour réaliser la table de composition des aliments propre aux aliments locaux, une liste complète des aliments et ingrédients consommés dans la zone d'étude a été établie. La composition nutritionnelle des aliments listés a été ajoutée à partir de la base de données de composition des aliments contenue dans le logiciel Word Food Dietary Assessment System.

\section{Entrée des données de consommation alimentaire}

Après l'élaboration de la table de composition des aliments, les données de consommation alimentaire des enfants ont été saisies à l'aide du logiciel Word Food Dietary Assessment System en utilisant la base de données mentionnée ci-dessus. L'usage de ce logiciel demande également l'entrée d'informations préliminaires telles que: l'identifiant, l'âge, le poids, le sexe et la date de l'enquête. A ces informations préliminaires, s'ajoutent les données de consommation alimentaire qui représentent la quantité de chaque aliment unique consommé par les enfants. Le logiciel a également permis d'obtenir les quantités journalières de 
nutriments apportés par la consommation alimentaire.

Les formules

$\checkmark \quad$ Estimation de la

contribution des aliments à base de manioc à l'apport en énergie et nutriments des aliments de complément et de l'alimentation des enfants de 6 à 35 mois

La contribution d'un aliment ou groupe d'aliments à l'apport en énergie et nutriments est estimée par le rapport entre la quantité de nutriment apporté par cet aliment et la quantité totale de nutriments apportée par les aliments de complément et l'alimentation des enfants de 6 à 35 mois. Pour le manioc et ses dérivés, la formule est la suivante :

$$
\text { ¿ } \mathrm{CMAJ}=\frac{\text { QNAAC }}{\text { QTNAAC }} * 100
$$

$\mathrm{CMAJ}=$ Contribution du manioc à l'apport journalier $(\%)$ QNAAC= Quantité de nutriments apportée par l'aliment consommé

QTNAAC $=$ Quantité totale de nutriments apportée par les aliments consommés

$\checkmark$ Estimation de la contribution des aliments à base de manioc à la couverture des besoins en énergie et nutriments des aliments de complément et de l'alimentation des enfants de 6 à 35 mois

La contribution d'un aliment ou groupe d'aliments au taux de couverture des besoins en énergie et nutriments est estimée en comparant les apports en énergie et nutriments de l'aliment ou du groupe d'aliments aux besoins en énergie et nutriments recommandés. Pour le manioc et ses dérivés, la formule est la suivante :



\section{Données socioéconomiques et culturelles}

Les données qualitatives collectées ont été groupées par acteur. Ces données ont été ensuite codifiées et entrées à l'aide du logiciel SPSS qui a servi de logiciel d'analyse. Des statistiques descriptives ont été calculées et des tests non paramétriques ( $\mathrm{T}$ de Kendall) ont été effectués afin de préciser les tendances dans les idées énumérées.

\section{Analyse statistique}

Les analyses statistiques effectuées concernent les statistiques descriptives et les tests de comparaison de moyenne. Le test $\mathrm{t}$ de Student a été utilisé pour comparer entre les garçons et les filles les valeurs moyennes des variables de l'étude. Pour comparer les valeurs moyennes des variables pour les trois tranches d'âge, l'analyse de variance (ANOVA) a été utilisée. L'analyse statistique a été effectuée à l'aide du logiciel SPSS 16. Les tests statistiques sont considérés significatifs pour des valeurs de $\mathrm{P}<0,05$.

\section{RESULTATS \\ Caractéristiques sociodémographiques des enfants enquêtés}

En fonction de leur âge, les sujets ont été répartis en trois catégories : la tranche de 6 à 11 mois, la tranche de 12 à 23 mois et la tranche de 24 à 35 mois (Tableau 1). Globalement, les filles et les garçons sont équitablement répartis dans l'échantillon tandis qu'on observe des disparités au niveau des tranches d'âge. L'âge moyen des enfants de l'échantillon est 19,5 $\pm 9,2$ mois avec une taille moyenne de $77,9 \pm 7,5 \mathrm{~cm}$. Le poids moyen des enfants de l'échantillon est de $9,8 \pm 2,0 \mathrm{~kg}$. Pour toutes les tranches d'âge, le poids moyen et la taille moyenne des garçons apparaissent légèrement supérieurs à ceux des filles; respectivement $10,1 \pm 1,9 \mathrm{~kg}$ contre $9,4 \pm 2,0 \mathrm{~kg}$ et $78,7 \pm 6,5 \mathrm{~cm}$ contre $77,0 \pm 8,4$ $\mathrm{cm}$. Mais, signalons qu'il y a une différence significative entre les trois tranches d'âge: pour l'âge, la taille et le poids des enfants $(\mathrm{P}<0,05)$. Au sein de chaque tranche d'âge, il n'y a pas de différence significative entre filles et garçons en ce qui concerne l'âge, le poids et la taille. 
Etat nutritionnel des enfants de 6 à 35 mois

Le Tableau 2 montre les Z-scores moyens des différents types de malnutrition des enfants de 6 à 35 mois. La comparaison de chaque indicateur par groupe d'âge montre que les Z-scores moyens de l'émaciation (P/T) ne sont pas différents pour les trois tranches d'âge. De même, il n'y a pas de différence statistique entre les tranches de 12-23 mois et de 24-35 mois pour les Z-scores moyens du retard de croissance (T/A) et de l'insuffisance pondérale (P/A). Par contre, les enfants âgés de 6-11 mois affichent des Z-scores moyens (T/A) et (P/A) statistiquement supérieurs à ceux des tranches de 12-23 mois et 24-35 mois $(\mathrm{P}<0,05)$. La comparaison de chaque indicateur selon le sexe et au niveau de chaque tranche d'âge révèle que les Z-scores moyens des trois types de malnutrition ne sont pas différents entre les garçons et les filles.

La Figure 1 montre la prévalence des différents types de malnutrition au sein des enfants de 6 à 35 mois par sexe. La prévalence globale de l'émaciation, du retard de croissance et de l'insuffisance pondérale est de $8,6 \%, 32,7 \%$ et $17,1 \%$ respectivement. La prévalence de l'insuffisance pondérale au sein des garçons n'est pas différente de celle des filles (17\%). La prévalence du retard de croissance semble plus élevée chez les garçons $(34 \%)$ que chez les filles $(31,4 \%)$. Il en est de même pour la prévalence de l'émaciation. Elle semble plus élevée chez les garçons $(11,3 \%)$.

\section{Principaux groupes d'aliments consommés par les enfants de 6 à 35 mois}

Nous avons effectué une étude de consommation alimentaire des tranches d'âge ciblées dans cette étude. Il est apparu que, en complément au lait maternel, plusieurs groupes d'aliments sont consommés par les enfants (Tableau 3). Les céréales représentent les aliments de base consommés par tous les enfants. Les légumineuses et noix de palme constituent la deuxième catégorie d'aliments les plus consommés après les céréales. Elles sont consommées par $91 \%$ des enfants. Les fruits et légumes sont également consommés par plus de $75 \%$ des enfants, alors que $52 \%$ des enfants consomment des aliments d'origine animale tels que les poissons, œuf, viande et fromage. Les racines et tubercules représentés essentiellement par les dérivés du manioc, sont consommés par $36 \%$ des enfants. Ces principaux groupes d'aliments sont consommées sous plusieurs formes. Les céréales et les légumineuses sont transformées en farines puis en bouillie ou pâte ou cuites directement (riz et légumineuse). Les racines et tubercules sont consommés sous formes de bouillie ou sous forme de farine accompagnée d'autres aliments/plats. Les principaux plats d'accompagnement des céréales sont la sauce tomate, la friture et les sauces légumes. Les légumes feuilles utilisées en l'occurrence sont les feuilles de manioc (Manihot esculenta Crantz) et les feuilles de crincrin (Corchorus olitorius). Les huiles utilisées pour la préparation des différentes sauces consommées par les enfants sont essentiellement l'huile de palme et l'huile d'arachide. Les poissons séchés sont parfois ajoutés aux sauces au cours de la préparation. Entre les principaux repas, certains fruits notamment l'orange, la mangue, la banane, l'ananas sont parfois consommés par les enfants.

\section{Contribution du manioc à l'alimentation et à la nutrition \\ Dérivés de manioc consommés, fréquence de consommation et forme de consommation \\ Le groupe racines et tubercules} représente l'un des principaux groupes d'aliments de base consommé par les enfants. $\mathrm{Au}$ sein de ce groupe, les aliments les plus consommés sont les dérivés du manioc, tels que le gari, la farine de cossette de manioc, le manioc bouilli et les beignets de manioc. 
Parmi ces dérivés du manioc, il ressort qu'au bout d'une semaine, le gari a été consommé au moins une fois par 97\% (96 enfants) des 106 enfants de l'échantillon contre 6 à $15 \%$ pour les autres dérivés.

Le Tableau 4 présente les nombres et proportion d'enfants ayant consommé des dérivés de manioc au cours de la journée d'enquête par observation avec pesée des aliments. Le gari reste le dérivé de manioc le plus consommé: il a été consommé en moyenne par $34 \%$ des enfants, contre presque pas de consommation pour les cossettes de manioc.

En raison du fait que le gari a été le dérivé le plus consommé par une proportion substantielle des enfants, seul ce dérivé (gari) a été considéré dans la suite du travail. Le niveau de consommation (quantité) du gari a augmenté proportionnellement par tranche d'âge. Globalement, la quantité de gari consommée par les enfants de 24-35 mois (146 g) est plus élevée que celle des enfants de 12-23 mois $(79,7 \mathrm{~g})$ et des enfants de 6 à 11 mois (48,3 g). Le gari est essentiellement consommé sous forme délayée dans l'eau avec ou sans sucre et avec ou sans arachide. Il sert également d'accompagnement au niébé bouilli et assaissonné. Enfin, les enfants le consomme sous forme de gari «gnignan»: gari mélangé à du jus de tomate ou simplement à de l'huile de noix de palme plus du sel.

\section{Contribution du gari aux apports en énergie et en nutriments}

Le Tableau 5 présente la contribution moyenne du gari aux apports journaliers en énergie et nutriments de l'alimentation de complément et de l'alimentation des enfants de 6 à 35 mois. Cette contribution est relativement faible pour toutes les tranches d'âge mais augmente avec l'âge. La contribution du gari aux apports journaliers en énergie est de 7\%. Quant aux protéines, fer, zinc, la contribution du gari varie respectivement de 2 à $5 \%, 4$ à $9 \%$ et de 3 à $7 \%$ pour les trois tranches d'âge. Pour la vitamine $\mathrm{A}$, sa contribution est pratiquement nulle, le gari étant pauvre en cette vitamine.

La contribution du gari aux taux de couverture des besoins en nutriments des enfants de 6 à 35 mois est faible. Le gari permet de couvrir 4 à $22 \%$ des besoins en énergie des enfants. Il contribue pour 2 à $14 \%$ de la couverture de leurs besoins en protéines. Les besoins recommandés en fer et en zinc pour les enfants de 6-11 mois, 12-23 mois et 24-35 mois sont couverts respectivement à hauteur de 1 à $9 \%$ et 1 à $5 \%$ par le gari (Tableau 6).

\section{Facteurs socioéconomiques et culturels affectant la consommation du manioc}

Les facteurs socioéconomiques et culturels qui favorisent la consommation des dérivés du manioc par les enfants sont au nombre de quatre (Tableau 7). Le manioc est produit localement dans la commune de Djidja et transformé en différents produits. Ces dérivés du manioc sont faciles à préparer soit par délayage à froid dans de l'eau, soit par malaxage avec de l'eau chaude (piron) ou à l'huile (gari gnignan). Le gari gnignan est donné aux enfants pour calmer leur faim en attendant que le repas principal soit prêt. Ils sont peu coûteux, ce qui facilite leur accessibilité financière. Nous n'avons relevé ni tabou, ni interdit dans la consommation du manioc. Quant aux facteurs limitants, nous pouvons citer: les problèmes d'indigestion causés par certains dérivés, en l'occurrence le lafun et les contraintes liées à la production de certains dérivés du manioc (pain, biscuit, boisson). Ces contraintes sont: le manque de matériel et d'équipement de transformation appropriés, qui vient en première position, l'absence de marché d'écoulement et le manque de formation. 
Tableau 1: Caractéristiques des sujets.

\begin{tabular}{llcccc}
\hline Tranche d'âge & Sexe & Effectif & Age (mois)* & Poids (kg)* & Taille (cm)* \\
\hline \multirow{2}{*}{6 - 11 mois } & Filles & $17(59 \%)$ & $8,6 \pm 1,6$ & $8,0 \pm 1,6$ & $69,6 \pm 6,3$ \\
& Garçons & $12(41 \%)$ & $8,3 \pm 1,7$ & $8,9 \pm 1,0$ & $72,3 \pm 3,9$ \\
& Total & $29(27 \%)$ & $8,5 \pm 1,6$ & $8,4 \pm 1,5$ & $70,7 \pm 5,5$ \\
\hline \multirow{2}{*}{12 - 23 mois } & Filles & $18(43 \%)$ & $18,5 \pm 3,6$ & $9,3 \pm 1,8$ & $76,8 \pm 5,2$ \\
& Garçons & $24(57 \%)$ & $17,9 \pm 4,5$ & $9,8 \pm 2,0$ & $77,5 \pm 5,0$ \\
& Total & $42(40 \%)$ & $18,2 \pm 4,1$ & $9,6 \pm 1,9$ & $77,2 \pm 5,0$ \\
\hline \multirow{2}{*}{24 - 35 mois } & Filles & $18(51 \%)$ & $30,6 \pm 4,2$ & $10,9 \pm 1,3$ & $84,3 \pm 6,1$ \\
& Garçons & $17(49 \%)$ & $29,7 \pm 4,1$ & $11,5 \pm 1,3$ & $85,0 \pm 3,9$ \\
& Total & $35(33 \%)$ & $30,2 \pm 4,1$ & $11,2 \pm 1,3$ & $84,7 \pm 5,1$ \\
\hline Toutes les tranches & Filles & $53(50 \%)$ & $19,5 \pm 9,6$ & $9,4 \pm 2,0$ & $77,0 \pm 8,4$ \\
d'âge $(6-35$ & Garçons & $53(50 \%)$ & $19,5 \pm 8,9$ & $10,1 \pm 1,9$ & $78,7 \pm 6,5$ \\
mois) & Total & $106(100 \%)$ & $19,5 \pm 9,2$ & $9,8 \pm 2,0$ & $77,9 \pm 7,5$ \\
\hline *Moyenne+Ecart type & & & & &
\end{tabular}

Tableau 2: Z-scores moyens par tranche d'âge.

\begin{tabular}{lccc}
\hline Tranches d'âge & \multicolumn{3}{c}{ Z-scores (Moyenne \pm Ecart type)* } \\
\cline { 2 - 4 } & Z-score P/T & Z-score T/A & Z-sore P/A \\
\hline 6 - 11 mois & $-0,1 \pm 1,2^{\mathrm{a}}$ & $0,1 \pm 2,2^{\mathrm{a}}$ & $-0,1 \pm 1,5^{\mathrm{a}}$ \\
12 - 23 mois & $-0,4 \pm 1,6^{\mathrm{a}}$ & $-1,4 \pm 1,3^{\mathrm{b}}$ & $-0,9 \pm 1,5^{\mathrm{b}}$ \\
24 - 35 mois & $-0,3 \pm 1,1^{\mathrm{a}}$ & $-1,9 \pm 1,3^{\mathrm{b}}$ & $-1,3 \pm 0,8^{\mathrm{b}}$ \\
Toutes les tranches d'âge (6 - 35 mois) & $-0,3 \pm 1,3$ & $-1,2 \pm 1,8$ & $-0,8 \pm 1,4$ \\
\hline
\end{tabular}

*Les valeurs dans les colonnes affectées de la même lettre ne sont pas significativement différentes au seuil de $5 \%$.

Tableau 3 : Principaux groupes d'aliments et proportion d'enfants les ayant consommés.

\begin{tabular}{llc}
\hline Groupes d'aliments & Aliments & $\begin{array}{c}\text { Nombre d'enfants (\%) ayant } \\
\text { consommé les aliments }\end{array}$ \\
\hline Céréales & Maïs, Riz, Blé & $106(100)$ \\
Légumineuses et Noix & Niébé, Autres légumineuses, Noix & $96(91)$ \\
Fruits et légumes & Légumes feuilles, fruits & $83(78)$ \\
Aliments d'origine animale & Euf, Poisson, Viande, Fromage & $55(52)$ \\
Racines et Tubercules & Manioc, autres racines (Igname) & $38(36)$ \\
Autres (sucreries) & Bonbon, sucette & $13(12)$ \\
\hline
\end{tabular}


Tableau 4: Nombre et proportion (\%)* d'enfants âgés de 6 à 35 mois ayant consommé les dérivés de manioc pendant la journée de l'enquête à Djidja $(n=106)$.

\begin{tabular}{lcccc}
\hline Dérivés & $\begin{array}{c}\mathbf{6} \text { à } \mathbf{1 1} \text { mois } \\
(\mathbf{n = 2 9})\end{array}$ & $\begin{array}{c}\mathbf{1 2} \text { à } \mathbf{2 3} \text { mois } \\
(\mathbf{n = 4 2})\end{array}$ & $\begin{array}{c}\mathbf{2 4} \text { à } \mathbf{3 5} \text { mois } \\
(\mathbf{n = 3 5})\end{array}$ & $\begin{array}{c}\mathbf{6} \text { à } 35 \text { mois } \\
(\mathbf{n = 1 0 6})\end{array}$ \\
\hline Gari & $6(21 \%)$ & $15(36 \%)$ & $15(43 \%)$ & $36(34 \%)$ \\
Farine de cossette de manioc & $0(0 \%)$ & $1(2 \%)$ & $0(0 \%)$ & $1(1 \%)$ \\
\hline
\end{tabular}

* proportion calculés sur la base de $\mathrm{n}=36$ représentant le nombre total d'enfants ayant consommé les dérivés du manioc.

Tableau 5 : Contribution du gari aux apports journaliers en énergie et nutriments.

\begin{tabular}{llccccc}
\hline Tranche d'âge & & Effectif & $\begin{array}{c}\text { \% } \\
\text { Energie }\end{array}$ & $\begin{array}{c}\text { \% } \\
\text { Protéine }\end{array}$ & \% Fer & $\begin{array}{c}\text { \% } \\
\text { Zinc }\end{array}$ \\
\hline \multirow{3}{*}{6 à 11 mois } & Filles & 17 & $1 \pm 5$ & $0 \pm 2$ & $1 \pm 3$ & $1 \pm 3$ \\
& Garçons & 12 & $8 \pm 15$ & $4 \pm 8$ & $8 \pm 14$ & $5 \pm 10$ \\
& Total & 29 & $4 \pm 11^{\text {a }}$ & $2 \pm 6$ & $4 \pm 10$ & $3 \pm 7$ \\
\hline \multirow{3}{*}{12 à 23 mois } & Filles & 18 & $5 \pm 11$ & $3 \pm 6$ & $4 \pm 9$ & $4 \pm 9$ \\
& Garçons & 24 & $10 \pm 16$ & $5 \pm 8$ & $9 \pm 14$ & $8 \pm 12$ \\
& Total & 42 & $8 \pm 14^{\mathrm{b}}$ & $4 \pm 7$ & $7 \pm 12$ & $6 \pm 11$ \\
\hline \multirow{2}{*}{24 à 35 mois } & Filles & 18 & $12 \pm 15$ & $6 \pm 8$ & $11 \pm 15$ & $9 \pm 11$ \\
& Garçons & 17 & $8 \pm 13$ & $4 \pm 6$ & $7 \pm 12$ & $6 \pm 9$ \\
\hline Toutes les tranches & Total & 35 & $10 \pm 14^{\mathrm{c}}$ & $5 \pm 7$ & $9 \pm 14$ & $7 \pm 10$ \\
d'âge (6 à 35 mois) & Total & 106 & $7 \pm 13$ & $4 \pm 7$ & $7 \pm 12$ & $6 \pm 10$ \\
\hline Les valeurs dans les colonnes affectées de différentes lettres sont significativement différentes au seuil de 5\%.
\end{tabular}

Tableau 6 : Contribution du gari aux taux de couverture des besoins en énergie et nutriments.

\begin{tabular}{llccccc}
\hline Tranche d'âge & & Effectifs & \% Energie & \% Protéine & \% Fer & \% Zinc \\
\hline \multirow{3}{*}{ 6 à 11 mois } & Filles & 17 & $2 \pm 11$ & $1 \pm 5$ & $1 \pm 3$ & $1 \pm 3$ \\
& Garçons & 12 & $5 \pm 10$ & $3 \pm 5$ & $1 \pm 2$ & $1 \pm 2$ \\
& Total & 29 & $4 \pm 10^{\mathrm{a}}$ & $2 \pm 5$ & $1 \pm 3$ & $1 \pm 2$ \\
\hline \multirow{2}{*}{$\mathbf{1 2}$ à 23 mois } & Filles & 18 & $6 \pm 15$ & $4 \pm 9$ & $3 \pm 6$ & $1 \pm 4$ \\
& Garçons & 24 & $15 \pm 29$ & $9 \pm 14$ & $6 \pm 11$ & $3 \pm 6$ \\
& Total & 42 & $11 \pm 24^{\mathrm{b}}$ & $7 \pm 14$ & $5 \pm 10$ & $3 \pm 5$ \\
\hline \multirow{2}{*}{$\mathbf{2 4}$ à 35 mois } & Filles & 18 & $32 \pm 44$ & $21 \pm 28$ & $13 \pm 20$ & $7 \pm 10$ \\
& Garçons & 17 & $11 \pm 17$ & $8 \pm 12$ & $5 \pm 8$ & $3 \pm 4$ \\
\hline $\begin{array}{l}\text { Toutes les } \\
\text { tranches d'âge } \\
\text { (6 à 35 mois) }\end{array}$ & Total & 35 & $22 \pm 35^{\mathrm{c}}$ & $14 \pm 23$ & $9 \pm 14$ & $5 \pm 8$ \\
\hline
\end{tabular}

\footnotetext{
*Les valeurs dans les colonnes affectées de différentes lettres sont significativement différentes au seuil de $5 \%$.
} 
Tableau 7: Hiérarchisation des raisons d'utilisation des dérivés de manioc dans l'alimentation des enfants par les mères d'enfants $(n=151)$.

\begin{tabular}{lcc}
\hline Raisons de consommation & Rang moyen & Ordre de classement $^{*}$ \\
\hline Revenu des ménages & 1,05 & 1 \\
Commodité & 1,95 & 2 \\
Disponibilité des dérivés de manioc & 3,18 & 3 \\
Facilité de préparation & 3,82 & 4 \\
\hline
\end{tabular}

* Test de concordance de Kendall, $P=0,000$

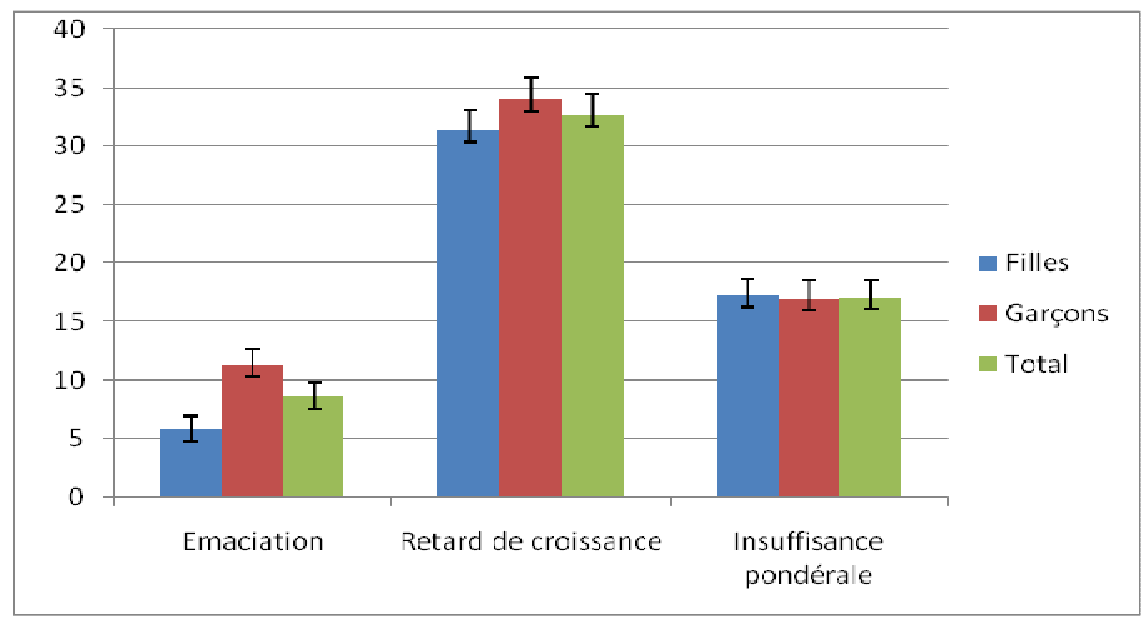

Figure 1: Prévalence des différents types de malnutrition.

\section{DISCUSSION}

\section{Situation nutritionnelle des enfants}

Les résultats des données anthropométriques ont montré que la malnutrition, surtout le retard de croissance des enfants de 6 à 35 mois constitue un problème de santé publique dans la commune de Djidja. En effet, en comparant les valeurs obtenues aux seuils de (<-3) définis par l'OMS (1995) pour évaluer la sévérité de la malnutrition dans une population d'enfants de 0-59 mois, il ressort que la prévalence du retard de croissance est élevée (>30\%). Le retard de croissance étant un processus cumulatif, la proportion d'enfants atteints augmente avec l'âge lorsqu'aucune intervention n'est faite (Allen et al., 2001). La prévalence de l'émaciation et de l'insuffisance pondérale estimée par la présente étude est supérieure à celle de (EDSB, 2006) qui est de $8 \%$ pour l'émaciation et de $14 \%$ pour l'insuffisance pondérale. Par contre, la prévalence de retard de croissance obtenue, est inférieure à celle de l'EDSB (2006) qui est de 43\%. Les données reportées par l'AGVSAN au Bénin en 2008 montre que la prévalence de l'émaciation, du retard de croissance, et de l'insuffisance pondérale chez les enfants de 6 à 59 mois est respectivement de 5\%,37\% (12\% sous forme sévère), et $17 \%$ ( $\mathrm{GdB}$ et al., 2009). Ces résultats semblent inférieurs à ceux obtenus lors de la présente étude dans le cas de l'émaciation. De même, la prévalence de 
l'émaciation et de l'insuffisance pondérale obtenue lors de la présente étude semble inférieure à celle obtenue par (Adomahou, 2009) qui est de $13 \%, 50 \%$ et $38 \%$ respectivement pour la prévalence de l'émaciation, du retard de croissance et de l'insuffisance pondérale. Pour l'émaciation, la prévalence de $8,6 \%$ obtenue serait due au fait, qu'au moment de l'enquête, il y avait eu un problème de santé au sein des enfants. La prévalence du retard de croissance et de l'insuffisance pondérale obtenue parait supérieure à celle estimée par (Honfo et al., 2005) où la prévalence était de $25 \%, 22 \%$ et $4 \%$ respectivement pour l'émaciation, le retard de croissance et l'insuffisance pondérale.

Bien que les milieux d'étude ou périodes d'évaluation soient différents, il ressort globalement de cette comparaison que la malnutrition sous forme d'émaciation, de retard de croissance et d'insuffisance pondérale est un phénomène général et persistant dans la commune de Djidja. De 2005 (Honfo et al., 2005) à 2010 (notre étude), il n'y a pas eu d'amélioration dans la situation nutritionnelle des nourrissons et des jeunes enfants de Djidja surtout dans le cas du retard de croissance et de l'insuffisance pondérale. Il est alors urgent d'identifier des stratégies novatrices visant à renforcer les interventions passées ou en cours. Il s'agit, entre autres, d'améliorer les apports nutritionnels des principaux groupes vulnérables à partir des aliments de base de la population et des ressources locales largement disponibles et facilement accessibles.

\section{Composition du régime alimentaire}

Les résultats de la présente étude ont également montré que le modèle alimentaire des enfants de 6 à 35 mois de l'échantillon est caractérisé par une contribution importante des céréales, suivie des légumineuses. Les fruits et légumes occupent une place relativement importante dans le régime de par la proportion d'enfants ayant consommés ce groupe d'aliments (78\%). Les racines et tubercules en général ont été consommés par un faible nombre d'enfants de l'échantillon (36\%). En considérant les enfants de 6 à 23 mois, $30 \%$ ont consommé des dérivés du manioc. Ce résultat n'est pas très différent de ceux obtenus dans le cadre de l'EDSB III, qui montrent que 29\% des enfants de 6 à 23 mois avaient consommé des produits à base de racines au cours des 24 heures précédent l'enquête. Les produits d'origine animale ont été également consommés par plus de la moitié des enfants. Ces aliments de base sont accompagnés de sauce de tomate ou de sauce de légume. L'huile de palme est celle la plus utilisée dans la préparation de ces différentes sauces. Ce type de régime s'apparente à ceux décrit au niveau national pour les enfants allaités ou non allaités. Il s'apparente également à ceux décrit lors de précédentes études par (Honfo et al., 2005) chez les enfants de 6 à 35 mois dans la commune de Djidja, par Adomahou (2009) dans la même commune et par Mitchikpè (2007) chez les enfants d'âge scolaire du Nord-Bénin. Ce type de régime dominé par les céréales est caractérisé par une faible teneur en micronutriments tels que le fer et le zinc.

\section{Place du manioc dans l'alimentation des enfants}

Selon les résultats de la présente étude, le gari reste le dérivé de manioc le plus consommé comme aliment de complément avec une fréquence de consommation d'au moins une fois par semaine, pour $91 \%$ des enfants. Le gari est un aliment obtenu à partir du manioc fermenté. Du point de vue composition nutritionnelle, il apporte une proportion importante d'énergie. Mais sa teneur en fer et en zinc est faible et il ne 
contient pas de vitamine A. Le gari contribue pour $7 \%$ aux apports en énergie, $4 \%$ à ceux des protéines et 6 à $7 \%$ à ceux des minéraux. Ces faibles contributions, ajoutées aux relativement faibles quantités journalières ingérées expliqueraient la faible contribution du gari à la couverture des besoins journaliers. Ainsi, en dehors de l'énergie pour toutes les tranches d'âge, la contribution du gari à la couverture des besoins journaliers en micronutriments essentiels comme le fer et le zinc est relativement faible ( 7 et $3 \%$ respectivement). Compte tenu de l'importance réduite du gari dans l'alimentation des enfants de 6 à 35 mois et compte tenu de ses valeurs nutritionnelles réduites, il ne serait pas le dérivé le plus approprié pour la promotion du manioc biofortifié dans l'alimentation de complément des enfants. Une raison qui renforcerait cette idée est aussi le fait que la transformation du manioc biofortifié en gari pourrait engendrée des pertes relativement élevées en vitamine A. Il serait donc plus approprié de promouvoir la consommation d'un autre dérivé du manioc demandant une transformation moins poussée tel que le manioc bouilli. Ceci ne serait pas si difficile étant donné que le manioc bouilli fait déjà partie des habitudes alimentaires de la plupart des régions du Bénin.

Facteurs socioéconomiques et culturels influençant la consommation des dérivés du manioc par les enfants de Djidja

L'étude qualitative des facteurs socioéconomiques et culturels déterminant la consommation des dérivés du manioc comme aliment de complément par les enfants a révélé que la disponibilité locale permanente (production et transformation), l'accessibilité financière du gari, sa commodité, de même que sa facilité et rapidité de préparation favorisent sa consommation plus ou moins régulière chez les enfants. Ceci constitue un atout important qui pourrait être valorisé, étant donné que l'une des conditions de durabilité des stratégies alimentaires telles que la biofortification des denrées de base pour la réduction de la malnutrition étant la disponibilité et l'accessibilité des produits biofortifiés.

Un autre facteur favorable en rapport avec la disponibilité permanente du gari est qu'il sert à calmer la faim (la commodité) des enfants en attendant le repas principal du soir. Ceci suggère que même s'il est consommé en petites quantités, du fait de la régularité de sa consommation, le manioc pourrait, en complément aux autres aliments, contribuer à une couverture des besoins journaliers. Cependant, une manière plus profitable de valoriser cet avantage serait de faire la promotion d'un dérivé du manioc autre que le gari pour une meilleure contribution à l'apport nutritionnel.

Enfin, la faiblesse du revenu a été également identifiée par les mères comme facteur les obligeant à donner du gari comme aliment de complément à leurs enfants. S'il n'est pas possible d'exercer une influence directe sur les conditions de vie des mères, il est toutefois possible d'améliorer la qualité de leur alimentation, de même que celle de leurs enfants. La biofortification semble une stratégie à moyen terme qui permettrait de renforcer la teneur en nutriments essentiels des aliments de base tels que le manioc. Dans le cas de la commune de Djidja, ceci pourrait être facilité par la disponibilité et l'accessibilité du manioc au niveau local, de même que l'existence des associations et groupements, des structures non gouvernementales d'appui et d'assistance, les centres de santé, qui par leur influence, faciliterait l'acceptation des produits biofortifiés dans la communauté. 


\section{Conclusion}

La situation nutritionnelle des enfants de 6 à 35 mois est préoccupante dans la commune de Djidja. La prévalence de l'émaciation et du retard de croissance est de $8,6 \%$ et $32,7 \%$ respectivement. Celle de l'insuffisance pondérale est de $17,1 \%$. Le gari est le dérivé de manioc le plus consommé par les enfants. Sa teneur en minéraux est relativement faible. La contribution du gari aux taux de couverture des besoins recommandé évolue avec l'âge, et est relativement faible pour les trois tranches d'âge. Sa contribution aux taux de couverture des besoins en vitamine A est pratiquement nulle. Ces résultats sont une conséquence de la faible contribution du gari aux apports journaliers en nutriments. Par ailleurs les facteurs socioéconomiques et culturels qui favorisent la consommation des dérivés du manioc par les enfants sont les revenus, la commodité du gari, la disponibilité et accessibilité permanente de ses dérivés et la facilité de préparation. On pourrait exercer une influence sur ces différents facteurs pour partir du manioc biofortifié afin d'améliorer l'apport nutritionnel du manioc et de ses dérivés au taux de couverture des besoins.

Eu égard aux résultats de cette étude, des actions soutenues doivent être faites pour assurer une bonne nutrition aux enfants.

\section{REMERCIEMENTS}

Les auteurs remercient l'Union Européenne à travers le projet INSTAPA (Grant agreement $n^{\circ} 211484$ ) pour son appui financier.

\section{REFERENCES}

Adjadogbedji CB. 2000. Les caractéristiques physico-chimiques des principales variétés de manioc en usage au Bénin: Relations avec la friabilité du produit après cuisson. Thèse d'Ingénieur
Agronome. Faculté des Sciences Agronomiques, Université National du Bénin.

Adomahou DGT. 2009. Analyse des pratiques d'alimentation et de l'état nutritionnel des enfants de 6-35 mois: Cas du village Kakatéhou/Ablédji (Commune de Djidja). Thèse Ingénieur Agronome. Option Nutrition et Sciences Alimentaires. Faculté des Sciences Agronomiques de l'Université d'Abomey-Calavi (FSA/UAC).

Allen LH, Gillespie SR. 2001. What Works? A Review of the Efficacy and Effectiveness of Nutrition Interventions. ACC/SCN nutrition policy paper $\mathrm{n}^{\circ} 19$, ADB Nutrition and development series n5. Philippines, p 124.

Chabi CLA. 2000. Etude de la production de Lafun au Bénin. Thèse d'Ingénieur Agronome. Abomey-Calavi, Bénin.

EDSB. 2006. Enquête Démographique et de Santé du Bénin. MCCAGPD/INSAE : Cotonou, Bénin, 354p.

FAO. 2001. The State of Food Insecurity in World: FAO: Rome; 27 p.

GDB, INSAE, PAM, UNICEF, FAO. 2009. Analyse Globale de la Vulnérabilité, de la Sécurité Alimentaire et de la Nutrition (AGVSAN) - République du Bénin (données de novembre et décembre 2008). Gouvernement du Bénin, Institut National de la Statistique et de l'Analyse Economique (Bénin), Programme Alimentaire Mondial, Fonds de Nations Unies pour l'Enfance et Organisation des Nations Unies pour l'alimentation et l'agriculture.

Gibson RS, Ferguson EL. 1999. An Interactive 24-Hour Recall for Assessing th Adequacy of Iron and Zinc Intakes in Develping Country. International life Science Institute : Washington, 175p. 
HarvestPlus. 2009. Améliorer les cultures pour une meilleure alimentation. HarvestPlus. 4p.

Honfo F, Dossa R, Hounhouigan J. 2005. Aflatoxin risk assessment, biological control option and interventions. Rapport d'enquête non publié 50p.

Mitchikpe CES. 2007. Towards a food-based approach to improve iron and zinc status of rural Beninese children: enhancing mineral bioavailability from sorghumbased food. Wageningen, Netherlands. $p$ 152.

Nago M, Hounhouigan J. 1998. La Transformation Alimentaire Traditionnelle des Racines et Tubercules au Bénin. CERNA, FSA/UAC : Bénin ; 97.

OMS. 1995. Utilisation et interprétation de l'anthropométrie. Rapport d'un comité d'experts. In MAIRE B, DELPEUCH F. (IRD-France), 2004. Indicateurs de nutrition pour le développement : Guide de référence. FAO, ROME, Italie; 69.

ONASA/CERPA ZOU. 2008. Département du zou/colline. Rapport d'Evaluation de la production Vivrière en 2008 et des perspectives alimentaires pour 2009 au Bénin: situation par département. Volume II, 187p.

Padonou SWG. 2009. Etude biochimique et microbiologique de la transformation du manioc (Manihot esculenta, Crantz) et qualité du Lafun produit au Bénin. Thèse pour obtenir le grade de Docteur de l'Université d'Abomey Calavi. 231p.

PAM/UNICEF/INSAE. 2009. Analyse globale de la vulnérabilité, de la sécurité alimentaire, de l'alimentation et de la nutrition (AGVSAN). Service de l'Analyse de la sécurité alimentaire du Programme Alimentaire Mondiale, Mai 2009.

RGPH. 2004. Recensement Général de la Population et de l'Habitat. MCCAGPD/INSAE: Cotonou, Bénin; 27. WHO. 2006. Child Growth Standards: Length/weight-for-age, Weight-for-age, Weight-for-Length, Weight-for-Height and Body Mass Index-for-Age Methods and Development. World Health Organization, Department of Nutrition for Health and Development: Geneva. 